E.Estrada,InstituteofMathematicsandApplications(IUMA),UniversidaddeZaragoza,

PedroCerbuna12,E-50009Zaragoza,Spain;estrada66@unizar.es 


\title{
"Melting" of complex networks. A mathematical model of complex networks resilience to external stress
}

\author{
Najlaa Alalwan ${ }^{1}$, Alex Arenas ${ }^{2}$ and Ernesto Estrada ${ }^{3-5}$ \\ ${ }^{1}$ Department of Mathematics and Statistics, University of Strathclyde, 26 Richmond Street, Glasgow G11HX, \\ UK. ${ }^{2}$ Departament d'Enginyeria Informàtica i Matemàtiques, Universitat Rovira $i$ Virgili, 43007 Tarragona, \\ Spain; ${ }^{3}$ Institute of Mathematics and Applications (IUMA), Universidad de Zaragoza, Pedro Cerbuna 12, \\ E-50009 Zaragoza, Spain; ${ }^{4}$ ARAID Foundation, Government of Aragón, 50018 Zaragoza, Spain.
}

\begin{abstract}
Complex networks are the representative graphs of interactions in many complex systems. Usually, these interactions are abstractions of the communication/diffusion channels between the units of the system. Recently we have proved analytically the existence of a universal phase transition in the communicability-a topological descriptor that reveals the efficiency of the network functionality in terms of these diffusive paths-of every simple network. This transition resembles the melting process occurring in solids. Here we study computationally this universal melting process in a large dataset of real-world networks and observe that the rate of melting of graphs changes either as an exponential or as a power-law with the inverse temperature representing the external stress to which the system is submitted to. At the local level we discover that the main driver for node melting is the eigenvector centrality of the corresponding node, particularly when the critical value of the inverse temperature approaches zero. That is, the most central nodes are the ones most at risk of triggering the melt down of the global network. These universal results can be used to sheds light on many dynamical diffusive-like processes on networks that present transitions as traffic jams, communication lost or failure cascades.
\end{abstract}

\section{Introduction}

The use of physical metaphors for studying complex networks is a very useful strategy to understand topological phenomena in terms of physical analogies. This includes, for instance, the use of theoretical tools developed in polymer physics, spin glass studies, Ising model 
simulations, discrete scaling and the theory of liquids to study complex systems. Therefore, the use of metaphors such as "phase transition", "quantum networks", "networks of harmonic oscillators", "vibrations on networks", etc. are among the most used and productive ways of exploring complex networks. These networks represent many physical, biological, social and engineering systems [ㅍ, $\mathbb{2}, \mathbf{3}]$ in which such physical analogies are not realizable in real-world scenarios.

In this context, we have recently developed a mathematical theory of topological melting of any graph and network [4]. This approach starts from the use of another metaphor, which is to consider a network as a general system of balls and springs submerged into a thermal bath at a given inverse temperature $\beta=\left(k_{B} T\right)^{-1}$ where $k_{B}$ is a constant [5]. Here the thermal bath represents the external stress to which the system is submitted to and $\beta$ represents a weight applied to every edge of the graph. The capacity of a node to transmit a perturbation at a given $\beta$ to another node is quantified by the thermal Green's function of the network [5]. Then, we have developed a model based on Lindemann criterion for melting at microscopic level [G]. According to Lindemann criterion [ [6, []], melting is caused by vibration instability in the crystal lattice, which eventually makes that the amplitude of vibration becomes so large that the atoms collide with their nearest neighbors, disturbing them and initiating the melting. Then, every substance is characterized by a melting point, which is the temperature at which such process starts. A crystal can be represented by a regular lattice [ 8$]$ in which atoms are the nodes and interactions between atoms are the edges of a simple graph. It is then easy to set up a vibrational model on this graph by considering it as a ball-and-spring system and studying the change of state in it as a result of raising the temperature using the Lindemann criterion [6]. Using such series of physical metaphors we have proved analytically the existence of a universal "melting" transition in the topology of a graph with the change of the parameter $\beta$.

The thermal Green's function used in this work is better known in the literature as the communicability function of a graph [9, 5]. It has found many applications in the analysis of real-world networks, such as in detecting changes in the contralesional hemisphere following 
strokes in humans [ㅍ], in the detection of symptoms of multiple sclerosis [П]], in the study of variants of epilepsy [1:2], in prediction of abnormal brain states [1.3], in early detection of Alzheimer's disease [14], in prediction of functional protein complexes [15], in the analysis of genetic diseases [[6], in the optimization of wireless networks [I7], in the evolution of granular

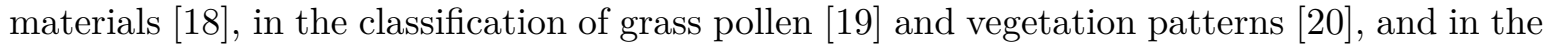
identification of the transcription factor critically involved with self-renewal of undifferentiated embryonic stem cells [피], to mention just a few of recent findings. Sensu stricto melting is the phase transition in which a solid is transformed into a liquid, and it is a fundamental physical process of elements, substances and materials, which results from the application of heat or pressure to the substance [22, 23$]$. Here, it is used as a way to characterize an abrupt transition in the communicability function between pairs of connected nodes in a network.

In this work we provide a new interpretation of this "algebraic" phenomenon occurring on network communicability when the parameter $\beta$ is changed systematically towards zero. We also study by the first time the existence and characteristics of this phenomenon in real-world networks. We discover here that the main driver for node melting is the eigenvector centrality of the corresponding node. That is, nodes with higher values of the Perron-Frobenious eigenvector melt at lower temperatures than those with smaller values of it. Thus, being "more central" according to the eigenvector centrality also indicates to be "more at risk" of triggering a "melt down" of the network communicability, which is an abrupt variation in the rate of change of this function with the parameter $\beta$.

\section{Mathematical model}

Here we describe a model for studying an analogous of the melting transition in networks. We consider simple, undirected graphs $\Gamma=(V, E)$ with $n$ nodes (vertices) and $m$ edges. In this case we consider that every node $i$ of the network is a ball of mass $m_{i}$ and that two nodes $i$ and $j$ are connected by a spring of spring constant $\omega_{i j}$. For the sake of simplicity we consider that all nodes are identical, i.e., $m_{i}=m, \forall i \in V$ and all springs are identical, i.e., $\omega_{i j}=\omega, \forall(i, j) \in E$. In order to avoid the translation movement of network os harmonic 
oscillators we tie every node to the ground with springs of constant $K \gg \max k_{i}$, where $k_{i}$ is the degree of the node $i$. We then consider that the position and the momentum of the system do not commute, which means that we consider the system as a network of quantum-harmonic oscillator (see [5] for details). The Hamiltonian describing the energy of this system is given by

$$
\hat{H}=\sum_{i} \hbar \Omega\left(a_{i}^{\dagger} a_{i}+\frac{1}{2}\right)-\frac{\hbar \omega^{2}}{4 \Omega} \sum_{i, j}\left(a_{i}^{\dagger}+a_{i}\right) A_{i j}\left(a_{j}^{\dagger}+a_{j}\right)
$$

where $A_{i j}$ are the elements of the adjacency matrix, $a_{i}^{\dagger}\left(a_{i}\right)$ are the annihilation (creation) operators, and $\Omega=\sqrt{K / m \Omega}$. We now consider that the network of quantum harmonic oscillators is submerged into a thermal bath with inverse temperature $\beta=\left(k_{B} T\right)^{-1}$, where $k_{B}$ is a constant and $T$ is the temperature. Then, it was previously proved that the thermal Green's function of this system is given by [5] :

$$
\tilde{G}_{p q}(\beta)=\exp (-\beta \hbar \Omega)\left(\exp \frac{\beta \hbar \omega^{2}}{2 \Omega} A\right)_{p q} .
$$

This function contains two parts, $\exp (-\beta \hbar \Omega)$ which is only rekated to physical parameters, and $\left(\exp \frac{\beta \hbar \omega^{2}}{2 \Omega} A\right)_{p q}$ which contains information about the topology of the network. Let us focus on the topology-dependent part of the thermal Green's function, which we will designate by

$$
G_{p q}(\beta)=(\exp (\beta A))_{p q}
$$

where we have set $\frac{\hbar \omega^{2}}{2 \Omega}=1$ for the sake of simplicity. This function $G_{p q}(\beta)$ is known as the network communicability function.

The term $e^{\beta \lambda_{1}} \psi_{1}(p) \psi_{1}(q)$ contributing to the communicability function represents the coordinated oscillation of all nodes in the graph due to the thermal bath at the corresponding value of $\beta$. Thus, we can obtain the difference between the total communicability and this term as 


$$
\begin{aligned}
\Delta G_{p q}(\beta)= & G_{p q}(\beta)-e^{\beta \lambda_{1}} \psi_{1}(p) \psi_{1}(q) \\
& {\left[\sum_{2 \leq j \leq n} e^{\beta \lambda_{j}} \psi_{j}^{+}(p) \psi_{j}^{+}(q)+\sum_{2 \leq j \leq n} e^{\beta \lambda_{j}} \psi_{j}^{-}(p) \psi_{j}^{-}(q)\right] } \\
& +\left[\sum_{2 \leq j \leq n} e^{\beta \lambda_{j}} \psi_{j}^{+}(p) \psi_{j}^{-}(q)+e^{\beta \lambda_{j}} \psi_{j}^{-}(p) \psi_{j}^{+}(q)\right] \\
= & \sum_{j \geq 2}^{\text {in-phase }} e^{\beta \lambda_{j}} \psi_{j}(p) \psi_{j}(q)-\left|\sum_{j \geq 2}^{\text {out }-o f-\text { phase }} e^{\beta \lambda_{j}} \psi_{j}(p) \psi_{j}(q)\right|
\end{aligned}
$$

where $\psi_{j}^{+}(p)\left(\psi_{j}^{-}(p)\right)$ means that the $p$ th entry of the $j$ th eigenvector is positive (negative) and we have used a nonincreasing ordering of the eigenvalues of $A$,i.e., $\lambda_{1}<\lambda_{2} \leq \cdots \leq \lambda_{n}$.

The "in-phase" term of the last expression corresponds to the case when both nodes have the same sign in the corresponding eigenvector, and the "out-of-phase" accounts for the cases in which the two nodes have different sign in the corresponding eigenvector. We notice that the second term is always negative and we use the modulus of it to express the term $\Delta G_{p q}(\beta)$ as a difference. $\Delta G_{p q}(\beta)$ accounts for the difference between the in- and out-of-phase vibrations of the corresponding pair of nodes.

Recently, Alawan et al. [4] have metaphorically considered the term

$$
M(\Gamma, \beta)=\max _{s \neq t \in V} \sum_{j=2}^{n} \psi_{j}(s) \psi_{j}(t) e^{\beta \lambda_{j}},
$$

as a threshold over which the vibration of two nodes in the network collapse to each other, producing the breaking of the edge connecting them. This is a metaphor imported from Lindeman theory of melting, such that the "melting" of a network starts in a when the vibrations of the nodes $p$ and $q$ at a given temperature measured by $\Delta G_{p q}(\beta)$ exceed the value of the maximum vibration of any pair of nodes in that graph at the same temperature, $M(\Gamma, \beta)$.

Mathematically, we implement this metaphor by defining the term 


$$
\Upsilon_{p q}(\beta)=M(\Gamma, \beta)+\Delta G_{p q}(\beta)
$$

such that when $\Upsilon_{p q}(\beta)<0$ the corresponding edge "melts". Thus, we construct a communicability graph $H\left(V, E^{\prime}, \beta\right)$ with the same set of vertices of the original one and edges given by

$$
A(H, \beta)_{p, q}=\left\{\begin{array}{l}
1 \text { if } \Upsilon_{p q}(\beta) \geq 0 \\
0 \text { if } \Upsilon_{p q}(\beta)<0
\end{array}\right.
$$

As shown in the work of Alawan et al. [4] this criterion is not enough for considering a melting process on networks. Such necassary and sufficient criterion is implemented through the definition of the so called Lindemann graph $F\left(V, E^{\prime \prime}, \beta\right)$ of $\Gamma$, which is the graph with the same set of vertices as $\Gamma$ and edge set defined by the following adjacency relation

$$
A(F, \beta)_{p, q}=\left\{\begin{array}{c}
1 \text { if }(p, q) \in E \text { and } \exists L_{p, q}, \\
0 \text { if }(p, q) \notin E \text { or } \nexists L_{p, q},
\end{array}\right.
$$

where $L_{p, q}$ are the so-called Lindemann path between the nodes $p$ and $q$ in $\Gamma$ at a given value of $\beta$, which are defined by the existence of a path connecting both nodes in the communicability graph $H\left(V, E^{\prime}, \beta\right)$ (not necessarily existing in the original graph). Using this framework, Alawan et al. [4] proved the following important result.

Theorem: Let $\Gamma=(V, E)$ be a non complete multipartite graph with communicability graph $H(\Gamma, \beta)$. Then, there is always exists a value $\beta_{c} \in[0, \infty)$ for which $H(\Gamma, \beta)$ is always connected for $\beta>\beta_{c}$ and always disconnected for $\beta \leq \beta_{c}$.

\section{Interpretation of the model}

In this section we provide a new interpretation of the phenomenon of graph melting based on the communicability. We should start by remarking that the idea of graph melting should be interpretaed as a physical metaphor for a completely mathematical phenomenon taking 
place on network communicability when the parameter $\beta$ is approaching asymptotically to zero. That is, in reality there is no disconnection of edges in the network at $\beta_{c}$, but also a significant change in the behavior of the communicability function for the different edges of the network. Here we explore by the first time what exactly is this change taking place on the communicability function.

The first thing that need to be considered here is that:

$$
G_{p, q}(0)=\left(e^{0 A}\right)_{p q}=I_{p q}=0
$$

Thus, because $G_{p, q}(\beta>0)>0$ we have a natural decreasing trend in the communicability when $\beta \rightarrow 0$. That is, when $\beta$ decreases, the communicability for any pair of nodes also decreases up to reaching the value of zero when $\beta=0$. Therefore, the main difference between one graph and another is in the rate at which the communicability decays as a function of $\beta$.

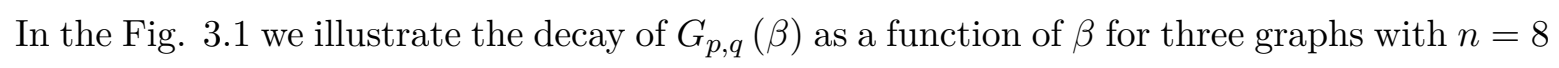
in which we have selected an edge from each of the graphs for calculating the communicability (there are no significant differences in selecting different edges of each graph). As can be seen the decay of the communicability with $\beta$ has two trends, for larger values of $\beta$ it follows an exponential decay and then a power-law. The power-law behavior starts, for these three graphs, at about $\beta=1$. If we select the straight line in the $\log -\log$ plot between $\beta=1$ and $\beta_{c}$, we can obtain the slope of this line by:

$$
m=\frac{\log G_{p q}(\beta=1)-\log G_{p q}\left(\beta_{c}\right)}{-\log \beta_{c}} .
$$

From which we can obtain the value of $\beta_{c}$

$$
-\log \beta_{c}=\frac{\log G_{p q}(\beta=1)-\log G_{p q}\left(\beta_{c}\right)}{m} .
$$

However, the obvious problem is that we ignore what is the value of $\beta_{c}$, so we cannot know what is the value of $G_{p q}\left(\beta_{c}\right)$ for a given graph. We notice for the three graphs considered here that $G_{p q}\left(\beta_{c}\right)$ is just a quadratic function of $G_{p q}(\beta=1)$ 


$$
G_{p q}\left(\beta_{c}\right) \approx 0.006761 G_{p q}^{2.117}(\beta=1)
$$

Thus, we can estimate $\beta_{c}$ from

$$
\beta_{c}=\exp \left[\frac{\log \left(0.006761 G_{p q}^{2.117}(\beta=1)\right)-\log G_{p q}(\beta=1)}{m}\right] .
$$

Therefore, the important remark here is that increasing $m$-the slope of the power-law decay of the communicability with $\beta$-increases $\beta_{c}$. That is, a large value of $m$ indicates a fast

decay of the communicability with $\beta$. When this happens, the graph melts at a relatively high value of $\beta_{c}$. On the other hand, if $m$ is small, e.g., close to zero, then the graph melts at a very low value of $\beta_{c}$. In order to interpret these results physically we again borrow a metaphor from the physics of melting. In this case we use the differences in melting between crystalline and amorphous materials. In the first case, i.e., crystalline materials, the melting occurs at a higher value of $\beta_{c}$ than for the amorphous materials. While in crystalline materials this phase gtransition is abrupt, in amorphous solids there is a very smooth transition from solid to liquid for a long range of temperatures. For instance, it is known that crystalline quartz melts at $1,550^{\circ} \mathrm{C}$, while amorphous quartz melts in the range $1,500-2,000^{\circ} \mathrm{C}$. Therefore, in those graphs resembling more a crystalline material, which have more regular structural patterns, the melting process occurs like in the case of $\Xi . \mathbb{( c )}$ with a more abrupt transition of the communicability with $\beta$ and at higher values of $\beta_{c}$. On the other hand, those graphs resembling more an amorphous solid, i.e., those having more irregular structural patterns, will have melting more similar to that of $\mathbf{B . D}(\mathrm{a})$ with a slow change of the communicability with $\beta$ and a smaller value of $\beta_{c}$.

\section{Datasets}

Here we consider a series of 47 complex networks arising from different scenarios. The datasets are described as follow:

Brain networks: Neurons: Neuronal synaptic network of the nematode C. elegans. In- 


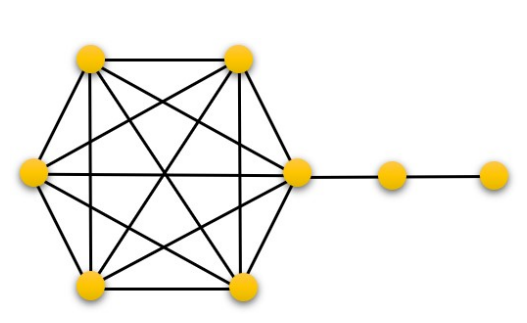

(a)

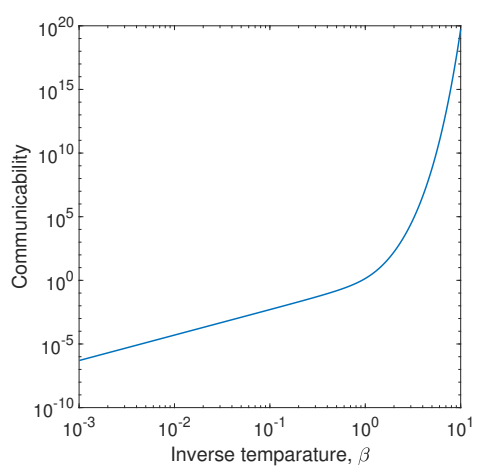

(d)

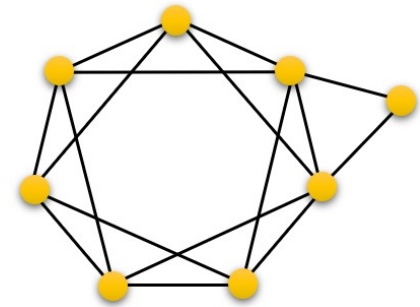

(b)

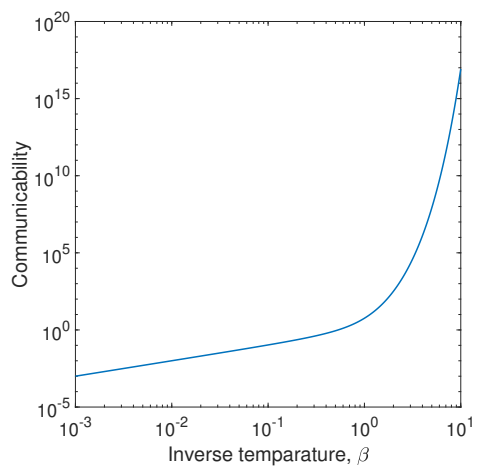

(e)

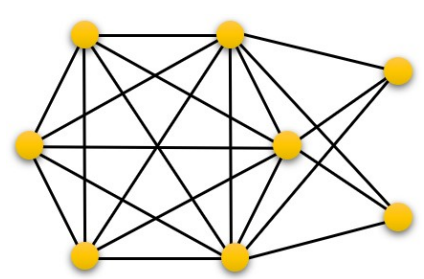

(c)

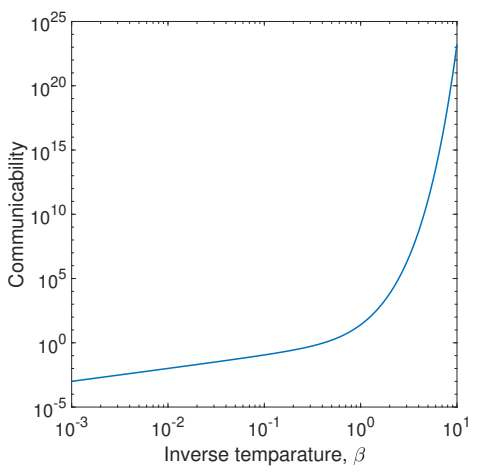

(f)

Figure 3.1: Illustration of the variation of $G_{p q}(\beta)$ with $\beta$ for a single edge (panels (d)-(f) in three different graphs with $n=8$ nodes illustrated in panels (a)-(c). The values of $\beta_{c}$ for the corresponding graphs are: 0.01 (a), 0.23 (b) and 0.75 (c). The plots are in log-log scale. 
cluded all data except muscle cells and using all synaptic connections [24]; Macaque visual cortices: the brain networks of macaque visual cortex and cat cortex, after the modifications introduced by Sporn and Kötter [25]. Ecological networks: Benguela: Marine ecosystem of Benguela off the southwest coast of South Africa [Z26]; Bridge Brook: Pelagic species from the largest of a set of 50 New York Adirondack lake food webs [27]; Canton Creek: Primarily invertebrates and algae in a tributary, surrounded by pasture, of the Taieri River in the South Island of New Zealand [ㅍ]; Chesapeake Bay: The pelagic portion of an eastern U.S. estuary, with an emphasis on larger fishes [2.9]; Coachella: Wide range of highly aggregated taxa from the Coachella Valley desert in southern California [30]; El Verde: Insects, spiders, birds, reptiles and amphibians in a rainforest in Puerto Rico [3]]; Little Rock: Pelagic and benthic species, particularly fishes, zooplankton, macroinvertebrates, and algae of the Little Rock Lake, Wisconsin, U.S. [32]; Reef Small: Caribbean coral reef ecosystem from the Puerto Rico-Virgin Island shelf complex [33]; Scotch Broom: Trophic interactions between the herbivores, parasitoids, predators and pathogens associated with broom, Cytisus scoparius, collected in Silwood Park, Berkshire, England, UK [34]; Shelf: Marine ecosystem on the northeast US shelf [35]; Skipwith: Invertebrates in an English pond [36]; St. Marks: Mostly macroinvertebrates, fishes, and birds associated with an estuarine seagrass community, Halodule wrightii, at St. Marks Refuge in Florida [37]; St. Martin: Birds and predators and arthropod prey of Anolis lizards on the island of St. Martin, which is located in the northern Lesser Antilles [38]; Stony Stream: Primarily invertebrates and algae in a tributary, surrounded by pasture, of the Taieri River in the South Island of New Zealand in native tussock habitat [39]; Ythan_1: Mostly birds, fishes, invertebrates, and metazoan parasites in a Scottish Estuary [40] ;Ythan_2: Reduced version of Ythan1 with no parasites [41]. Dolphins: social network of frequent association between 62 bottlenose dolphins living in the waters off New Zealand [42]. Informational networks: Roget: Vocabulary network of words related by their definitions in Roget's Thesaurus of English. Two words are connected if one is used in the definition of the other [43]; Small World: Citation network of papers that cite S. Milgram's 1967 Psychology Today paper or use Small World in title [44]. Biological networks: 
Protein-protein interaction networks in: Kaposi sarcoma herpes virus (KSHV) [45]; P. falciparum (malaria parasite) [46]; A. fulgidus [47]; H. pylori [48]; E. coli [49] and B. subtilis [50]; Trans_E.coli: Direct transcriptional regulation between operons in Escherichia coli [24, 51]; Trans_sea_urchin: Developmental transcription network for sea urchin endomesoderm development. [[1]]; Trans_yeast: Direct transcriptional regulation between genes in Saccaromyces cerevisae. [24, [1]]. Social and economic networks: Corporate: American corporate elite formed by the directors of the 625 largest corporations that reported the compositions of their boards selected from the Fortune 1000 in 1999 [52]; Prison: Social network of inmates in prison who chose "What fellows on the tier are you closest friends with?" [53]; Drugs: Social network of injecting drug users (IDUs) that have shared a needle in the last six months [54]; Zachary: Social network of friendship between members of the Zachary karate club [55]; Social3: Social network among college students in a course about leadership. The students choose which three members they wanted to have in a committee [56]; High_Tech: Friendship ties among the employees in a small high-tech computer firm which sells, installs, and maintain computer systems [57, 58]; Saw Mills: Social communication network within a sawmill, where employees were asked to indicate the frequency with which they discussed work matters with each of their colleagues [5.9, 58]; World trade: World trade network of miscellaneous manufacture of metals (MMM) in 1994 [58]. Technological and infrastructural networks: Electronic: Three electronic sequential logic circuits parsed from the ISCAS89 benchmark set, where nodes represent logic gates and flip-flop [24]; USAir97: Airport transportation network between airports in US in 1997 [44]. Software networks: Collaboration networks associated with six different open-source software systems, which include collaboration graphs for three Object Oriented systems written in $\mathrm{C}++$, and call graphs for three procedural systems written in C. The class collaboration graphs are from version 4.0 of the VTK visualization library; the CVS snapshot dated 4/3/2002 of Digital Material (DM), a library for atomistic simulation of materials; and version 1.0.2 of the AbiWord word processing program. The call graphs are from version 2.4.19 of the kernel of the Linux operating system, version 3.23.32 of the MySQL relational database system, and version 1.2.7 of the XMMS multimedia system. Details of the 
construction and/or origin of these networks are provided in Myers [60]].

\section{Results and Discussion}

We split our analysis into two parts. First we consider global properties of the networks and then we analyze the influence of node-level centrality on the melting process of these networks.

\subsection{Global analysis}

We start here by finding the value of $\beta$ at which the transition between connected to disconnected Lindemann graph occurs. Our first task is to relate the values of $\beta_{c}$ to some simple topological parameters of the networks in order to understand the structural dependence of this transition. With this goal we study the following structural representative parameters of networks described in Table 1:

Table 1: Global structural parameters of networks used in this work

\begin{tabular}{|c|c|c|c|c|}
\hline No. & Index & Formula & Observations & Ref. \\
\hline 1 & Edge density & $\delta=\frac{2 m}{n(n-1)}$ & $\begin{array}{c}m \text { is the number of edges and } n \\
\text { is the number of nodes }\end{array}$ & {$[\Pi]$} \\
\hline 2 & Average degree & $\bar{k}=\frac{1}{n} \sum_{i=1}^{n} k_{i}=\frac{2 m}{n}$ & $\begin{array}{l}k_{i} \text { is the degree of the node } i, \\
\text { i.e., the number of adjacent } \\
\text { nodes to } i \text {. }\end{array}$ & {$[\Pi]$} \\
\hline
\end{tabular}




\begin{tabular}{|c|c|c|c|c|}
\hline 3 & $\begin{array}{l}\text { Maximum } \\
\text { degree }\end{array}$ & $k_{\max }=\max _{i} k_{i}$ & $\begin{array}{l}\text { Ain connected graph with } n>2 \\
2 \leq k_{\text {max }} \leq n-1\end{array}$ & {$[\mathbf{I}]$} \\
\hline 4 & $\begin{array}{l}\text { Average } \\
\text { clustering } \\
\text { coefficient }\end{array}$ & $\bar{C}=\frac{1}{n} \sum_{i=1}^{n} \frac{2 t_{i}}{k_{i}\left(k_{i}-1\right)}$ & $\begin{array}{l}t_{i} \text { is the number of triangles } \\
\text { incident to the vertex } i \text { : } \\
\qquad t_{i}=\frac{1}{2}\left(A^{3}\right)_{i i}\end{array}$ & [6]] \\
\hline 5 & $\begin{array}{l}\text { Average path } \\
\text { length }\end{array}$ & $\bar{l}=\frac{1}{n(n-1)} \sum_{i<j} d(i, j)$ & $\begin{array}{l}\text { A measure of the } \\
\text { 'small-worldness' of the network. }\end{array}$ & {$[\Pi]$} \\
\hline 6 & $\begin{array}{c}\text { Shortest path } \\
\text { efficiency }\end{array}$ & $E=\sum_{i<j} 1 / d(i, j)$ & $\begin{array}{c}\text { Also known as the Harary index } \\
\text { of a graph }\end{array}$ & $\begin{array}{l}{[62,} \\
63]\end{array}$ \\
\hline 7 & $\begin{array}{c}\text { Largest } \\
\text { eigenvalue of } \\
A\end{array}$ & $\lambda_{1}$ & $\begin{array}{c}\lambda_{1}>\lambda_{2} \geq \cdots \geq \lambda_{n}, \text { it represents } \\
\text { a sort of average degree in the } \\
\text { network. }\end{array}$ & {$[\Pi]$} \\
\hline 8 & $\begin{array}{l}\text { Second largest } \\
\text { eigenvalue of } \\
A\end{array}$ & $\lambda_{2}$ & & {$[\bar{T}]$} \\
\hline
\end{tabular}




\begin{tabular}{|c|c|c|c|c|}
\hline 9 & Spectral gap & $\Delta=\lambda_{1}-\lambda_{2}$ & $\begin{array}{l}\text { A quantity related to } \\
\text { isoperimetric properties of } \\
\text { graphs. A large spectral gap } \\
\text { indicates the lack of structural } \\
\text { bottlenecks in the network. }\end{array}$ & {$[\Pi]$} \\
\hline 10 & $\begin{array}{l}\text { Average com- } \\
\text { municability } \\
\text { distance }\end{array}$ & $\bar{\xi}=\frac{\sum_{p<q}\left(G_{p p}+G_{q q}-2 G_{p q}\right)^{1}}{n(n-1)}$ & $\begin{array}{c}\text { A measure of the average } \\
\text { quality of communication in a } \\
\text { network, where } \\
G_{p q}=\sum_{j=1}^{n} \varphi_{j, p} \varphi_{j, q} \exp \left(\lambda_{j}\right) \text { is } \\
\text { the communicability between } \\
\text { the corresponding nodes. }\end{array}$ & $\begin{array}{l}{[64,} \\
65]\end{array}$ \\
\hline 11 & $\begin{array}{l}\text { Average } \\
\text { resistance } \\
\text { distance }\end{array}$ & $\bar{\Omega}=\sum_{i<j} \Omega_{i j}$ & $\begin{array}{c}\text { A measure related to hitting } \\
\text { and commute times in a random } \\
\text { walk on the network, where } \\
\varrho_{i j}=\sum_{k=2}^{n} \frac{1}{\mu_{k}}\left(\psi_{k, i}-\psi_{k, j}\right)^{2}\end{array}$ & {$[66]$} \\
\hline 12 & $\begin{array}{l}\text { Average com- } \\
\text { municability } \\
\text { angle }\end{array}$ & $\bar{\theta}=\frac{\sum_{p<q} \cos ^{-1}\left(\frac{G_{p q}}{\sqrt{G_{p p} G_{q q}}}\right)}{n(n-1)}$ & $\begin{array}{l}\text { A measure of spatial efficiency } \\
\text { of a network. } 0 \leq \bar{\theta} \leq 90 \text {, where } \\
\text { the lower bound indicates high } \\
\text { spatial efficiency and the upper } \\
\text { one indicates a poor spatial } \\
\text { efficiency. }\end{array}$ & {$[67]$} \\
\hline
\end{tabular}

We investigate correlations between these measures (see Table $\nabla$ ) and the values of $\beta_{c}$ for the 47 networks studied here in linear, semi-log and logarithmic scales. The most significant correlation was obtained for $\ln \beta_{c}$ and $\ln \delta(r=0.79)$, where $r$ is the Pearson correlation coefficient. Also significant are the correlations between $\ln \beta_{c}$ and $\bar{l}(r=-0.72)$, and with $\ln E(r=0.72)$.

The correlations found for $\ln \beta_{c}$ with some of the previous structural parameters may 


\begin{tabular}{|c|c|c|c|c|c|c|c|c|c|c|c|}
\hline network & $n$ & $m$ & $\bar{k}$ & $k_{\max }$ & $\bar{C}$ & $\bar{l}$ & $E$ & $\lambda_{1}$ & $\lambda_{2}$ & $\varrho$ & $\beta_{c}$ \\
\hline Skipwith & 35 & 353 & 20.17 & 32 & 0.63 & 1.42 & 0.79 & 22.08 & 3.43 & 1.583 & $2.9 \cdot 10^{-2}$ \\
\hline Social3 & 32 & 80 & 5.00 & 13 & 0.33 & 2.30 & 0.51 & 5.97 & 3.81 & 1.582 & $2.2 \cdot 10^{-2}$ \\
\hline Macaque & 32 & 194 & 12.13 & 22 & 0.65 & 1.66 & 0.69 & 14.04 & 7.33 & 1.569 & $1.8 \cdot 10^{-2}$ \\
\hline Zackary & 34 & 78 & 4.59 & 17 & 0.57 & 2.41 & 0.49 & 6.72 & 4.98 & 1.698 & $1.2 \cdot 10^{-2}$ \\
\hline World Trade & 80 & 875 & 21.88 & 77 & 0.75 & 1.72 & 0.64 & 30.13 & 10.61 & 2.042 & $8.4 \cdot 10^{-3}$ \\
\hline Reef Small & 50 & 503 & 20.12 & 39 & 0.61 & 1.60 & 0.70 & 23.75 & 8.58 & 1.771 & $7.4 \cdot 10^{-3}$ \\
\hline St. Martin & 44 & 218 & 9.91 & 27 & 0.33 & 1.93 & 0.59 & 12.53 & 6.97 & 1.745 & $7.1 \cdot 10^{-3}$ \\
\hline Coachella & 30 & 241 & 16.07 & 25 & 0.71 & 1.46 & 0.77 & 18.15 & 5.09 & 1.530 & $6.7 \cdot 10^{-3}$ \\
\hline St. Marks & 48 & 218 & 9.08 & 19 & 0.28 & 2.09 & 0.55 & 11.86 & 4.93 & 1.797 & $5.7 \cdot 10^{-3}$ \\
\hline Benguela & 29 & 191 & 13.17 & 24 & 0.57 & 1.62 & 0.72 & 15.23 & 4.080 & 1.525 & $4.0 \cdot 10^{-3}$ \\
\hline PIN KSHV & 50 & 114 & 4.56 & 16 & 0.13 & 2.84 & 0.42 & 7.41 & 3.61 & 1.910 & $3.6 \cdot 10^{-3}$ \\
\hline Shelf & 81 & 1451 & 35.83 & 69 & 0.59 & 1.57 & 0.72 & 41.92 & 11.72 & 1.977 & $3.5 \cdot 10^{-3}$ \\
\hline PIN A. fulgidus & 32 & 36 & 2.25 & 9 & 0.06 & 3.60 & 0.35 & 3.50 & 2.77 & 1.698 & $3.2 \cdot 10^{-3}$ \\
\hline Sawmill & 36 & 62 & 3.44 & 13 & 0.31 & 3.14 & 0.40 & 4.97 & 3.27 & 1.716 & $3.0 \cdot 10^{-3}$ \\
\hline Hitech & 33 & 91 & 5.52 & 16 & 0.45 & 2.36 & 0.51 & 7.94 & 4.08 & 1.677 & $3.0 \cdot 10^{-3}$ \\
\hline Ythan2 & 92 & 416 & 9.04 & 50 & 0.22 & 2.25 & 0.49 & 15.77 & 6.14 & 2.205 & $2.7 \cdot 10^{-3}$ \\
\hline Prison & 67 & 142 & 4.24 & 11 & 0.31 & 3.35 & 0.36 & 5.59 & 4.62 & 1.946 & $1.6 \cdot 10^{-3}$ \\
\hline Ythan1 & 134 & 593 & 8.85 & 65 & 0.23 & 2.40 & 0.46 & 16.74 & 7.46 & 2.404 & $1.6 \cdot 10^{-3}$ \\
\hline Chesapeake & 33 & 71 & 4.30 & 10 & 0.20 & 2.80 & 0.45 & 5.74 & 4.53 & 1.644 & $1.3 \cdot 10^{-3}$ \\
\hline Stony & 112 & 830 & 14.82 & 45 & 0.07 & 2.34 & 0.49 & 22.70 & 6.28 & 2.234 & $9.9 \cdot 10^{-4}$ \\
\hline Small World & 233 & 994 & 8.53 & 147 & 0.56 & 2.37 & 0.45 & 20.96 & 14.72 & 2.758 & $9.1 \cdot 10^{-4}$ \\
\hline PIN B. subtilis & 84 & 98 & 2.33 & 17 & 0.04 & 4.05 & 0.29 & 4.75 & 3.77 & 2.233 & $8.3 \cdot 10^{-4}$ \\
\hline Canton & 108 & 707 & 13.09 & 47 & 0.05 & 2.35 & 0.49 & 19.56 & 7.04 & 2.208 & $6.2 \cdot 10^{-4}$ \\
\hline Little Rock & 181 & 2318 & 25.61 & 105 & 0.35 & 2.22 & 0.51 & 40.82 & 26.17 & 2.460 & $4.2 \cdot 10^{-4}$ \\
\hline Dolphins & 62 & 159 & 5.13 & 12 & 0.26 & 3.36 & 0.38 & 7.19 & 5.94 & 1.939 & $1.8 \cdot 10^{-4}$ \\
\hline PIN Malaria & 229 & 604 & 5.28 & 35 & 0.17 & 3.38 & 0.33 & 9.78 & 7.92 & 2.628 & $1.6 \cdot 10^{-4}$ \\
\hline Electronic1 & 122 & 189 & 3.10 & 10 & 0.06 & 4.93 & 0.25 & 4.11 & 3.63 & 2.209 & $1.1 \cdot 10^{-4}$ \\
\hline Trans Urchin & 45 & 80 & 3.56 & 14 & 0.21 & 3.22 & 0.39 & 6.68 & 2.95 & 1.927 & $9.0 \cdot 10^{-5}$ \\
\hline Neurons & 280 & 1973 & 14.09 & 77 & 0.28 & 2.63 & 0.42 & 23.29 & 14.07 & 2.665 & $9.0 \cdot 10^{-5}$ \\
\hline Bridge Brook & 75 & 542 & 14.45 & 41 & 0.20 & 2.17 & 0.54 & 20.64 & 12.70 & 2.030 & $6.0 \cdot 10^{-5}$ \\
\hline El Verde & 156 & 1439 & 18.45 & 83 & 0.21 & 2.30 & 0.50 & 31.49 & 9.41 & 2.425 & $5.3 \cdot 10^{-5}$ \\
\hline Electronic2 & 252 & 399 & 3.17 & 14 & 0.06 & 5.81 & 0.20 & 4.36 & 3.96 & 2.540 & $3.6 \cdot 10^{-5}$ \\
\hline Scotch Broom & 154 & 366 & 4.75 & 36 & 0.14 & 3.39 & 0.33 & 14.71 & 6.23 & 2.678 & $1.8 \cdot 10^{-5}$ \\
\hline PIN H. pyroli & 710 & 1396 & 3.93 & 55 & 0.02 & 4.15 & 0.26 & 10.46 & 8.25 & 3.276 & $6.0 \cdot 10^{-6}$ \\
\hline Electronic3 & 512 & 819 & 3.20 & 22 & 0.05 & 6.86 & 0.17 & 5.01 & 4.12 & 2.904 & $3.9 \cdot 10^{-6}$ \\
\hline Software Digital & 150 & 198 & 2.64 & 25 & 0.05 & 4.85 & 0.25 & 6.702 & 4.84 & 2.581 & $3.0 \cdot 10^{-6}$ \\
\hline Roget & 994 & 3640 & 7.32 & 28 & 0.15 & 4.08 & 0.27 & 12.03 & 9.81 & 3.213 & $3.0 \cdot 10^{-6}$ \\
\hline Software_VTK & 771 & 1357 & 3.52 & 83 & 0.06 & 4.53 & 0.24 & 11.45 & 8.71 & 3.400 & $2.0 \cdot 10^{-6}$ \\
\hline Software Abi & 1035 & 1719 & 3.32 & 89 & 0.06 & 5.08 & 0.22 & 11.94 & 7.68 & 3.571 & $2.0 \cdot 10^{-6}$ \\
\hline USAir97 & 332 & 2126 & 12.81 & 139 & 0.63 & 2.74 & 0.41 & 41.23 & 17.31 & 3.029 & $1.6 \cdot 10^{-7}$ \\
\hline Trans Yeast & 662 & 1062 & 3.21 & 71 & 0.05 & 5.20 & 0.22 & 9.98 & 8.45 & 3.314 & $2.0 \cdot 10^{-8}$ \\
\hline Corporate elite & 1586 & 11540 & 14.55 & 65 & 0.50 & 3.51 & 0.31 & 23.23 & 19.23 & 3.403 & $2.0 \cdot 10^{-8}$ \\
\hline PIN E. coli & 230 & 695 & 6.04 & 36 & 0.22 & 3.78 & 0.31 & 15.93 & 8.57 & 2.783 & $1.7 \cdot 10^{-8}$ \\
\hline Trans E. coli & 328 & 456 & 2.78 & 72 & 0.11 & 4.83 & 0.25 & 9.06 & 6.30 & 3.029 & $9.0 \cdot 10^{-9}$ \\
\hline Drugs & 616 & 2012 & 6.53 & 58 & 0.55 & 5.28 & 0.23 & 18.01 & 14.23 & 3.230 & $1.8 \cdot 10^{-9}$ \\
\hline Software XMMS & 971 & 1802 & 3.71 & 36 & 0.05 & 6.35 & 0.18 & 10.28 & 8.93 & 3.430 & $9.0 \cdot 10^{-10}$ \\
\hline Software MySQL & 1480 & 4190 & 5.66 & 220 & 0.16 & 5.47 & 0.23 & 21.71 & 14.41 & 3.754 & $2.0 \cdot 10^{-14}$ \\
\hline
\end{tabular}

Table 2: Values of some of the global structural parameters studied here for the set of real-world neworks analyzed as well as the values of the critical $\beta$ at which the melting of these networks occur. Networks are 
be hiding something about the real structural characteristic of networks that influence their "melting". For instance, the negative correlation between edge density and $\beta_{c}$ seems suspicious. Our intuition tells us that, under all other structural conditions the same, high density networks should melt at higher temperatures, i.e., lower $\beta_{c}$, than lower density ones. This is exactly what it is observed in molecular crystals of nonpolar molecules, such as linear alkanes $[68]$. Then, the fact that smaller and denser (real-world) networks are the ones having the largest $\beta_{c}$, i.e., they have Lindemann graphs easier to disconnect, may indicate that the "degree homogeneity" of these networks more than their sizes or densities is the real driver of their melting. In order to capture these degree irregularities we recall the definition of the average degree of a network

$$
\bar{k}=\frac{2 m}{n}=\frac{\overrightarrow{1}^{T} A \overrightarrow{1}}{\overrightarrow{1}^{T} \overrightarrow{1}}
$$

The right-hand side of the previous equation is useful to think that the spectral radius of the adjacency matrix is a sort of average degree, which instead of counting only the number of nearest neighbors of a node consider also a more global picture around it

$$
\lambda_{1}=\frac{\vec{\psi}_{1}^{T} A \vec{\psi}_{1}}{\vec{\psi}_{1}^{T} \vec{\psi}_{1}} .
$$

Notice that $\bar{k} \leq \lambda_{1}$ with equality if and only if the graph is regular. Thus, the term $\left(\lambda_{1} / \bar{k}\right)$ represents the ratio of a more global environment of a node to its more local one. That is, the ratio $\left(\lambda_{1} / \bar{k}\right)$ indicates how a node "sees" as average its global environment in relation to its nearest neighbors. In a regular graph its local environment, i.e., its degree, is identical to the degree of its neighbors, second neighbors, and so on and we get that $\left(\lambda_{1} / \bar{k}\right)=1$. Then, we can define the following index of global to local degree heterogeneity

$$
\varrho(G)=n\left(\frac{\lambda_{1}}{\bar{k}}\right)
$$

Notice that $\varrho(G)=\left(\frac{\lambda_{1}}{\delta}\right)$, which may explain the previously observed correlation between $\ln \beta_{c}$ and $\ln \delta$. We have then used $\varrho(G)$ as an indicator of the global to local heterogeneity of 


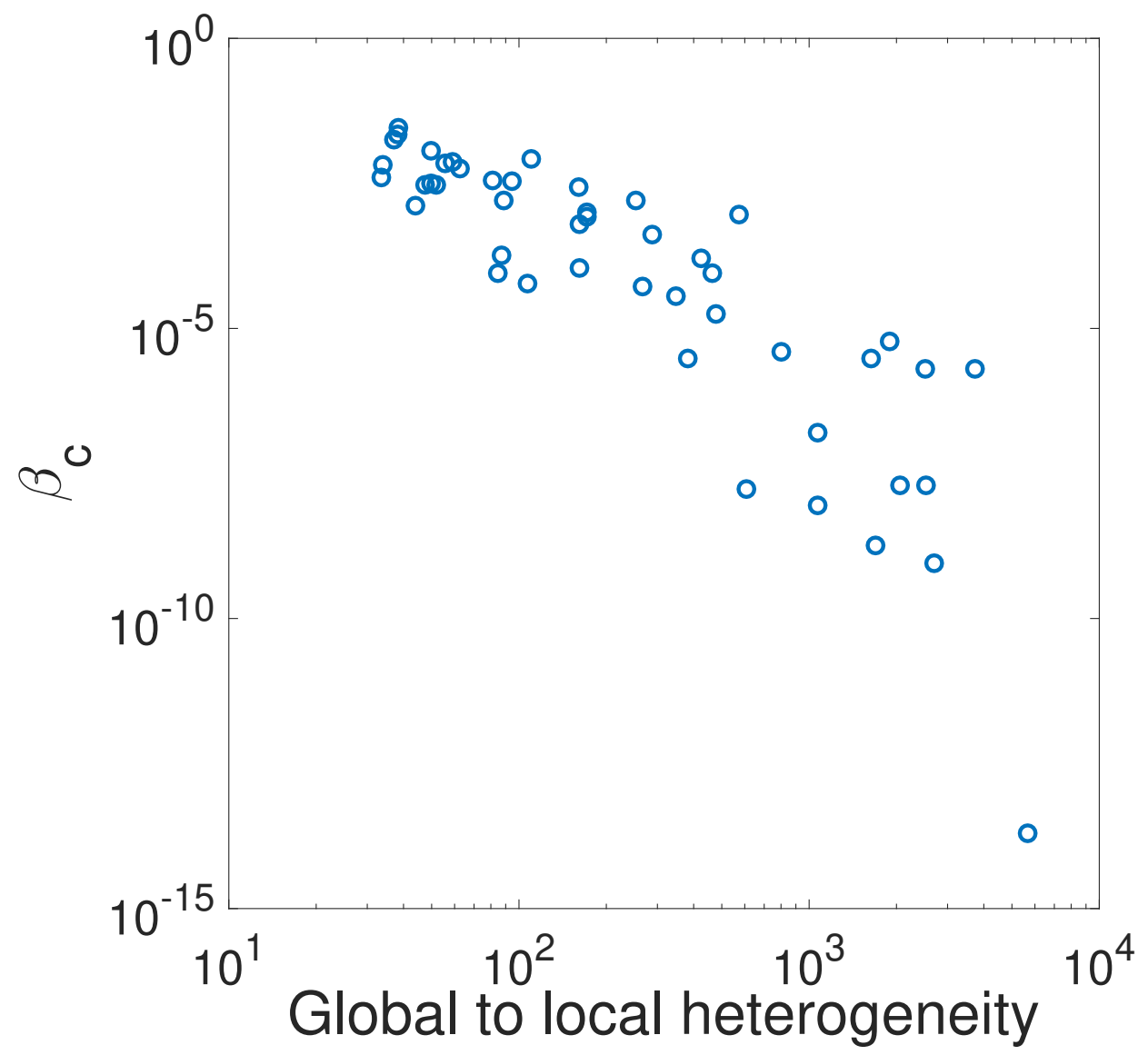

Figure 5.1: Changes of $\beta_{c}$ for 47 real-world networks as a function of their global/local degree heterogeneity as described in this work.

the 47 real-world networks analyzed here. In Fig. 5.] we illustrate the log-log plot of $\varrho(G)$ versus $\beta_{c}$, which has correlation coefficient $r=-0.85$.

The most important message of this section is the following. The disconnection of the Lindemann graph of a given graph, i.e., its melting, depends very much on the differences between global and local degree heterogeneities. Regular graphs are easier to melt than nonregular ones, and the more irregular-in terms of global to local degree heterogeneity-the graph is the smallest the value of $\beta_{c}$, i.e., more difficult to melt.

\subsection{Local analysis}

In this subsection we are interested in the local analysis of the effects of decreasing the value of $\beta$ on the topological structure of a network. In particular we investigate computationally two important aspects of the graph melting process: (i) How the nodes of a network melt? 


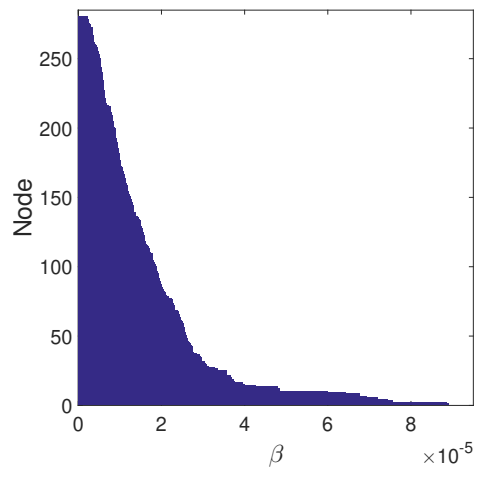

(a)

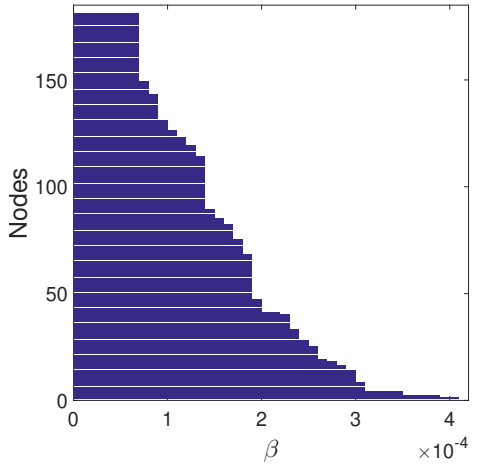

(b)

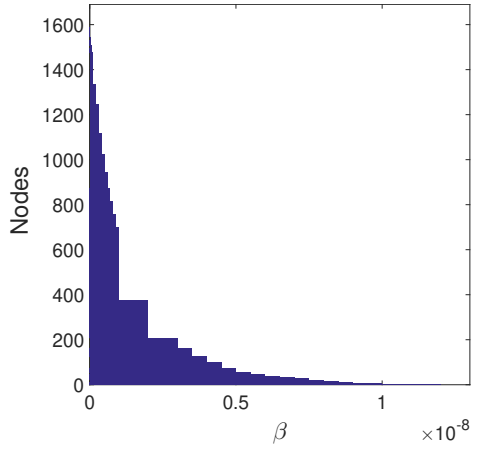

(c)

Figure 5.2: Illustration of the melting barcodes of the networks of neurons (a), Little Rock (b) and corporate elite (c).

and (ii) Which structural parameter drives the melting of the nodes? For investigating these questions we consider a subset of the real-world networks studied in this work. We create a melting barcode plot in which we plot every node in the $y$-axis and in the $x$-axis we provide the value of $\beta$ at which the corresponding node disconnect from the giant connected component of the graph. In Fig. 52 we illustrate the melting barcodes of three networks: neurons (a), Little Rock (b) and corporate elite (c). We need to read these melting barcodes from right to left as the melting process starts at higher values of $\beta$ and proceed by decreasing it. There are significant differences in the three barcodes presented which point out to the differences existing in the melting processes of the different graphs analyzed. First, we can observe that the shape of the melting barcodes are different. While in "neurons" the decay resembles an exponential curve, in "Little Rock" it is almost linear and in the "corporate elite" it displays a more skewed shape (see further for quantitative analysis). In the second place, the barcodes of "Little Rock" and of corporate elite display regions in which large groups of nodes are disconnected at the same temperature, while in neurons the change is smoother.

We then investigate the rate of change of the melting process in the networks analyzed by considering the shape of the histogram of the number of nodes "melted" at a given temperature. That is, we construct the histograms of the number of nodes melted in a temperature range versus the range of temperatures. In general we observe two kinds of decay of the number of nodes melted at a given temperature in relation to the inverse temperature. They 


\begin{tabular}{|c|c|c|c||c|c|c|c|}
\hline \multirow{2}{*}{ network } & \multicolumn{3}{|c|}{ Eq. 5.4 } & \multirow{2}{*}{ network } & \multicolumn{3}{|c|}{ Eq. 5.5 } \\
\cline { 2 - 5 } & $a$ & $\zeta$ & $r^{2}$ & & $b$ & $\gamma$ & $r^{2}$ \\
\hline \hline Prison & 24.58 & $-1.858 \cdot 10^{-3}$ & 0.790 & Macaque & $5.59 \cdot 10^{-15}$ & -6.953 & 0.756 \\
\hline Neurons & 178 & $-6.032 \cdot 10^{-4}$ & 0.975 & Stony & $4.84 \cdot 10^{-12}$ & -3.515 & 0.887 \\
\hline Small World & 334.9 & $-1.06 \cdot 10^{-4}$ & 0.995 & PIN B. subtilis & $2.34 \cdot 10^{-3}$ & -1.016 & 0.976 \\
\hline Ythan & 91.82 & $-3.61 \cdot 10^{-3}$ & 0.965 & Roget & $5.40 \cdot 10^{-8}$ & -1.441 & 0.992 \\
\hline Electronic 1 & 75.87 & $-5.99 \cdot 10^{-4}$ & 0.934 & Software_Abi & $7.05 \cdot 10^{-17}$ & -2.621 & 0.999 \\
\hline PIN H. pylori & 1233 & $-2.89 \cdot 10^{-6}$ & 0.999 & Corporate elite & $9.96 \cdot 10^{-14}$ & -1.744 & 0.999 \\
\hline
\end{tabular}

Table 3: Values of the fitting parameters for the Eqs. 5.4 and 5.5 displaying the relation between the number of nodes melted at a given value of $\beta$ as a function of $\beta$ for several real-world networks.

are:

$$
\eta=a \exp (\zeta \cdot \beta)
$$

$$
\eta=b \cdot \beta^{\gamma},
$$

where $\eta$ is the number of nodes melted at a given value of $\beta$. For some of the smallest networks it was not possible to find any particular law of the decay of $\eta$ as a function of $\beta$. These were the cases of the networks of Benguela $(n=29)$, Coachella $(n=30)$, Social3 $(n=32)$, St. Marks $(n=48)$, as well as for the network of Little Rock, which is not so small $(n=181)$ but it also has a very disperse histogram. For the rest of the networks analyzed we display the parameters of the fitting to Eqs. 5.4 and 5.5 in Table $\mathbf{3}$.

The fitting parameters given in Table [ indicate the differences in the rates of melting of the networks analyzed. These rates of melting represent a new measure of the robustness of networks to the effects of external stresses to which the networks are submitted to, as accounted for by the inverse temperature. For instance, those networks melting according to Eq. 5.4 are more robust to external stresses than the ones melting according to Eq. 5.5. In comparing those networks that melt exponentially with $\beta$ it is clear that the social network of inmates in prison (Prison) and the food web of Ythan are significantly less robust to such external stresses than the protein interaction network of H. pylori. The network representing the visual cortex of macaque melts very quickly in relation to the rest of the 
networks analyzed indicating that once the external stress has trigger the melting process the nodes of this network disconnect very fast from the giant connected component.

Finally, we investigate which structural parameters determine the melting process of the nodes of a network. In particular we consider here the role of node centrality on the melting of the corresponding node. We then analyze the relation between the value of $\beta$ at which a node melts and its degree centrality (DC), $k_{i}$, i.e., the degree of the node $i$; the closeness centrality, (CC): $C C_{i}=1 / \sum_{j \neq i} d(i, j)$, where $d(i, j)$ is the shortest path distance from $i$ to every other node of the graph [69]; the betweenness centrality, (BC): $B C_{i}=\sum_{r \neq i \neq s} \rho(r, i, s) / \sum_{r \neq s} \rho(r, s)$, where $\rho(r, i, s)$ is the number of shortest paths between $r$ and $s$ that pass over node $i$, and $\rho(r, s)$ is the total number of shortest paths between $r$ and $s\left[6.9,[0]\right.$; the eigenvector centrality, $E C_{i}$ : the $i$ th entry of the eigenvector $\vec{\psi}_{1}$ corresponding to the largest eigenvalue $\lambda_{1}$ of the adjacency matrix [[T]; the subgraph centrality, $S C_{i}$ : the $i$ th diagonal entry of $\exp (A)$ [[r2]. In general, we observe that the values of $\beta$ at which the nodes melt correlate very well with EC. All networks studied displayed Pearson correlation coefficients between these two parameters higher than 0.90 , with the exceptions of the networks of Benguela and Macaque visual cortex. In addition we investigate the coefficient of variation $(\mathrm{CV})$ of the values of $\beta$ at which a node melts estimated from a linear regression with EC. This coefficient is given by the standard deviation of the estimate divided by the mean of the values of $\beta$ at which the nodes melt in a given network. Here we provide the values of both Pearson correlation coefficient and CV in percentage for the networks investigated: Benguela $(r=0.68,34.3 \%)$, Coachela $(r=0.93,15.1 \%)$, Social3 $(r=0.95,16.2 \%)$, Macaque $(r=0.82$, $19.8 \%)$, St. Marks $(r=0.97,11.7 \%)$, Prison $(r=0.998,3.9 \%)$, PIN B. subtilis $(r=0.999$, $3.4 \%)$, Stony $(r=0.94,13.8 \%)$, Electronic1 $(r=0.99999,0.4 \%)$, Ythan1 $(r=0.99,8.6 \%)$, Small World $(r=0.998,5.0 \%)$, Little Rock $(r=0.989,6.9 \%)$, Neurons $(r=0.999,1.3 \%)$, Roget $(r=0.997,7.6 \%)$, PIN H. pylori $(r=0.997,7.6 \%)$, Software Abi $(r=0.996,19.7 \%)$, Corporate elite $(r=0.99,18.1 \%)$. An important point to have into account here is that although the correlation coefficients are in general very high, the values of CV indicate that the correlations are characterized by certain levels of dispersion. For instance, the networks 
of Software Abi and the Corporate elite have CV close to $20 \%$ although they have correlation coefficients larger than 0.99 .

In general, we observe that when $\beta_{c}$ is arbitrarily small, the correlation between the value of $\beta$ at which the node melts and EC is better than when $\beta_{c}$ is relatively large, e.g. Benguela, Coachela, Social3, Macaque, St. Marks, etc. The reason for that difference is the following. Let us recall that at $\beta_{c}$ the value of the Lindemann criterion is negative, that is

$$
\Delta \tilde{G}_{p q}\left(\beta_{c}\right)=\sum_{j=2}^{n} \psi_{j}(p) \psi_{j}(q) e^{\beta \lambda_{j}}<0
$$

Let $\beta_{c}$ be arbitrarily small such that we have $e^{\beta_{c} \lambda_{j}} \approx 1$ for all $j$ and

$$
\begin{aligned}
-\left|\Delta \tilde{G}_{p q}\left(\beta_{c}\right)\right| & =M\left(\Gamma, \beta_{c}\right)+\sum_{j=2}^{n} \psi_{j}(p) \psi_{j}(q) \\
& =M\left(\Gamma, \beta_{c}\right)-\psi_{1}(p) \psi_{1}(q),
\end{aligned}
$$

where $M\left(\Gamma, \beta_{c}\right)$ is obviously a constant. Then, if we take the sum of all the values of $\Delta \tilde{G}_{p q}\left(\beta_{c}\right)$ for the node $p$ we have

$$
-\sum_{q \neq p}\left|\Delta \tilde{G}_{p q}\left(\beta_{c}\right)\right|=M\left(\Gamma, \beta_{c}\right)-\psi_{1}(p) \sum_{q \neq p} \psi_{1}(q)
$$

which clearly explains the observed high positive correlation between the values of $\beta$ at which a node melts and $\psi_{1}(p)$ for networks having $\beta_{c}$ very close to zero. Also, it explains why those networks for which $\beta_{c}$ is not sufficiently small display bad correlations between the values of $\beta$ at which a node melts and $\psi_{1}(p)$.

This result has important consequences for the robustness of networks. Those networks displaying a high robustness to external stresses, such that $\beta_{c}$ is very close to zero, start their melting by the most central nodes according to EC. That is, if we consider a network like the USA transportation network, which has $\beta_{c}$ of the order of $10^{-7}$, we will observe that the first 


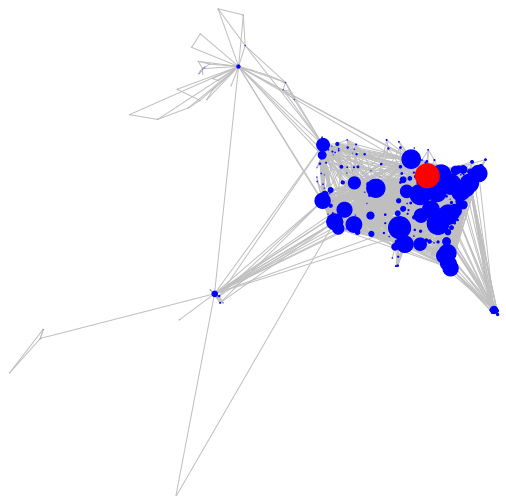

(a)

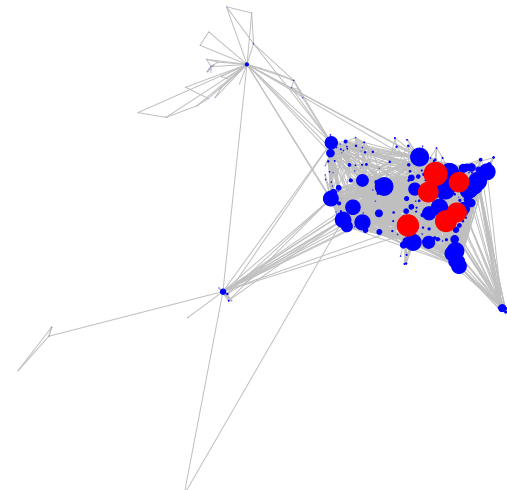

(b)

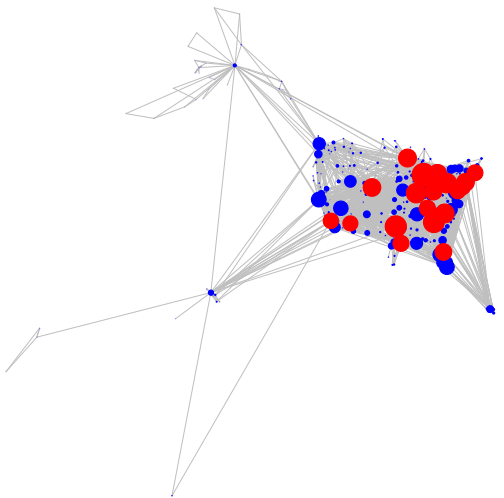

(c)

Figure 5.3: Snapshots of the melting process of the USAir97 network at three different values of $\beta$, namely at1.5 $10^{-7}(\mathrm{a}), 1.25 \cdot 10^{-7}(\mathrm{~b}), 1.0 \cdot 10^{-7}(\mathrm{c})$.

airports to be disconnected from the giant connected component are the most important ones in terms of their connectivity. Here we give the list of the first airports separated from the giant connected component in order of their disconnection: Chicago O'Hare, Dallas/Forth Worth Int., The William B. Hartsfield (Atlanta), Detroit Metropolitan, Pittsburgh Intel., Lambert-St. Louis, Charlotte/Douglas Int. (see Fig. 5.3).

\section{Conclusions and future outlook}

The analysis of a series of real-world networks has given us the possibility of exploring the global and local structural characteristics of networks which drives their melting. At the global topological level, we have shown here that the value $\beta_{c}$ at which the melting of a network occurs depends mainly on the differences between the local and global degree heterogeneities existing in the graph. At the local one we have observed that the melting is triggered by the nodes having the higher eigenvector centrality in the network, particularly in those cases where the melting temperature is very close to zero. This means that "being too central is dangerous" as it may trigger a catastrophic melt down of the network.

The analysis of networks melting as proposed here opens many new possibilities for the study of network robustness to external stresses. There are many mathematical and computational questions that remain open from the current study. They include, but are not 
limited to, the following ones: (i) A more exhaustive analysis of the topological (global and local) drivers of the network melting; (ii) How certain specific network characteristics, e.g., clustering, modularity, degree assortativity, etc., influence the melting temperature and the melting process of artificial and real-world networks?; (iii) Is there a ranking of certain classes of networks, e.g., "small-world", "scale-free" etc., according to their melting? We hope the reader can help to answer some of these questions and generate new ones that clarify our understanding of network robustness to external stresses.

\section{Acknowledgement}

The authors thank Dr. F. Arrigo, and Prof. D. H. Higham for useful comments and suggestions which improve the presentation of the material. NA thanks Iraqui Government for a Doctoral Fellowship at the University of Strathclyde.

\section{References}

[1] E. Estrada, The structure of complex networks: theory and applications, Oxford University Press, 2012.

[2] E. Estrada, Graph and network theory, Mathematical Tools for Physicists, Wiley.

[3] M. E. Newman, The structure and function of complex networks, SIAM review 45 (2) (2003) 167-256.

[4] N. Alalwan, A. Arenas, E. Estrada, Topological melting in networks of granular materials, Journal of Mathematical Chemistry 57 (3) (2019) 875-894.

[5] E. Estrada, N. Hatano, M. Benzi, The physics of communicability in complex networks, Physics reports 514 (3) (2012) 89-119.

[6] F. A. Lindemann, The calculation of molecular eigen-frequencies, Phys. Z.,(West Germany) 11 (14) (1910) 609-612. 
[7] Z. Jin, P. Gumbsch, K. Lu, E. Ma, Melting mechanisms at the limit of superheating, Physical Review Letters 87 (5) (2001) 055703.

[8] S. R. Phillpot, S. Yip, D. Wolf, How do crystals melt?, Computers in physics 3 (6) (1989) $20-31$.

[9] E. Estrada, N. Hatano, Communicability in complex networks, Physical Review E 77 (3) (2008) 036111.

[10] J. J. Crofts, D. J. Higham, R. Bosnell, S. Jbabdi, P. M. Matthews, T. Behrens, H. Johansen-Berg, Network analysis detects changes in the contralesional hemisphere following stroke, Neuroimage 54 (1) (2011) 161-169.

[11] Y. Li, V. Jewells, M. Kim, Y. Chen, A. Moon, D. Armao, L. Troiani, S. Markovic-Plese, W. Lin, D. Shen, Diffusion tensor imaging based network analysis detects alterations of neuroconnectivity in patients with clinically early relapsing-remitting multiple sclerosis, Human brain mapping 34 (12) (2013) 3376-3391.

[12] I. M. Campbell, M. Rao, S. D. Arredondo, S. R. Lalani, Z. Xia, S.-H. L. Kang, W. Bi, A. M. Breman, J. L. Smith, C. A. Bacino, et al., Fusion of large-scale genomic knowledge and frequency data computationally prioritizes variants in epilepsy, PLoS Genet 9 (9) (2013) e1003797.

[13] Y. Iturria-Medina, Anatomical brain networks on the prediction of abnormal brain states, Brain connectivity 3 (1) (2013) 1-21.

[14] M. Mancini, M. A. De Reus, L. Serra, M. Bozzali, M. P. Van Den Heuvel, M. Cercignani, S. Conforto, Network attack simulations in alzheimer's disease: The link between network tolerance and neurodegeneration, in: Biomedical Imaging (ISBI), 2016 IEEE 13th International Symposium on, IEEE, 2016, pp. 237-240.

[15] X. Ma, L. Gao, Predicting protein complexes in protein interaction networks using a core-attachment algorithm based on graph communicability, Information Sciences 189 (2012) 233-254. 
[16] I. M. Campbell, R. A. James, E. S. Chen, C. A. Shaw, Netcomm: a network analysis tool based on communicability, Bioinformatics (2014) btu536.

[17] H. Chan, L. Akoglu, Optimizing network robustness by edge rewiring: a general framework, Data Mining and Knowledge Discovery 30 (5) (2016) 1395-1425.

[18] D. M. Walker, A. Tordesillas, Topological evolution in dense granular materials: a complex networks perspective, International Journal of Solids and Structures 47 (5) (2010) $624-639$.

[19] L. Mander, M. Li, W. Mio, C. C. Fowlkes, S. W. Punyasena, Classification of grass pollen through the quantitative analysis of surface ornamentation and texture, Proceedings of the Royal Society of London B: Biological Sciences 280 (1770) (2013) 20131905.

[20] L. Mander, S. C. Dekker, M. Li, W. Mio, S. W. Punyasena, T. M. Lenton, A morphometric analysis of vegetation patterns in dryland ecosystems, Open Science 4 (2) (2017) 160443.

[21] B. D. MacArthur, A. Sevilla, M. Lenz, F.-J. Müller, B. M. Schuldt, A. A. Schuppert, S. J. Ridden, P. S. Stumpf, M. Fidalgo, A. Maayan, et al., Nanog-dependent feedback loops regulate murine embryonic stem cell heterogeneity, Nature cell biology 14 (11) (2012) $1139-1147$.

[22] R. W. Cahn, Melting and the surface, Nature 323 (6090) (1986) 668-669.

[23] V. Alexiades, Mathematical modeling of melting and freezing processes, CRC Press, 1992.

[24] R. Milo, S. Shen-Orr, S. Itzkovitz, N. Kashtan, D. Chklovskii, U. Alon, Network motifs: simple building blocks of complex networks, Science 298 (5594) (2002) 824-827.

[25] O. Sporns, R. Kötter, Motifs in brain networks, PLoS Biol 2 (11) (2004) e369.

[26] P. Yodzis, Local trophodynamics and the interaction of marine mammals and fisheries in the benguela ecosystem, Journal of Animal Ecology 67 (4) (1998) 635-658. 
[27] G. A. Polis, Complex trophic interactions in deserts: an empirical critique of food-web theory, The American Naturalist 138 (1) (1991) 123-155.

[28] C. Townsend, Disturbance, resource supply, and food-web architecture in streams, Ecology letters 1 (1998) 200-209.

[29] R. R. Christian, J. J. Luczkovich, Organizing and understanding a winters seagrass foodweb network through effective trophic levels, Ecological modelling 117 (1) (1999) 99-124.

[30] P. H. Warren, Spatial and temporal variation in the structure of a freshwater food web, Oikos (1989) 299-311.

[31] D. P. Reagan, R. B. Waide, The food web of a tropical rain forest, University of Chicago Press, 1996.

[32] K. Havens, Scale and structure in natural food webs., Science(Washington) 257 (5073) (1992) 1107-1109.

[33] S. Opitz, Trophic interactions in Caribbean coral reefs, Vol. 1085, WorldFish, 1996.

[34] J. Memmott, N. Martinez, J. Cohen, Predators, parasitoids and pathogens: species richness, trophic generality and body sizes in a natural food web, Journal of Animal Ecology 69 (1) (2000) 1-15.

[35] J. Link, Does food web theory work for marine ecosystems?, Marine ecology progress series $230(2002) 1-9$.

[36] P. Yodzis, Diffuse effects in food webs, Ecology 81 (1) (2000) 261-266.

[37] L. Goldwasser, J. Roughgarden, Construction and analysis of a large caribbean food web, Ecology 74 (4) (1993) 1216-1233.

[38] N. D. Martinez, Artifacts or attributes? effects of resolution on the little rock lake food web, Ecological monographs 61 (4) (1991) 367-392. 
[39] D. Baird, R. E. Ulanowicz, The seasonal dynamics of the chesapeake bay ecosystem, Ecological monographs 59 (4) (1989) 329-364.

[40] M. Huxham, S. Beaney, D. Raffaelli, Do parasites reduce the chances of triangulation in a real food web?, Oikos (1996) 284-300.

[41] S. Hall, D. Raffaelli, Food-web patterns: lessons from a species-rich web, The Journal of Animal Ecology (1991) 823-841.

[42] D. Lusseau, K. Schneider, O. J. Boisseau, P. Haase, E. Slooten, S. M. Dawson, The bottlenose dolphin community of doubtful sound features a large proportion of longlasting associations, Behavioral Ecology and Sociobiology 54 (4) (2003) 396-405.

[43] Roget's thesaurus of english words and phrases, project gutenberg (2002). URL http://www.gutenberg.org/etxt/22

[44] V. Batagelj, A. Mrvar, Analysis of large networks, 2006.

[45] P. Uetz, Y. Dong, C. Zeretzke, C. Atzler, A. Baiker, B. Berger, S. Rajagopala, M. Roupelieva, D. Rose, E. Fossum, J. Haas, Herpesviral protein networks and their interaction with the human proteome (2006).

[46] D. LaCount, M. Vignali, R. Chettier, A. Phansalkar, R. Bell, J. Hesselberth, L. Schoenfeld, I. Ota, S. Sahasrabudhe, C. Kurschner, S. Fields, A protein interaction network of the malaria parasite plasmodium falciparum (2005).

[47] M. Motz, I. Kober, C. Girardot, E. Loeser, U. Bauer, M. Albers, G. Moeckel, E. Minch, H. Voss, C. Kilger, M. Koegl, Elucidation of an archaeal replication protein network to generate enhanced pcr enzymes, Journal of Biological Chemistry 277 (18) (2002) 1617916188.

[48] C.-Y. Lin, C.-L. Chen, C.-S. Cho, L.-M. Wang, C.-M. Chang, P.-Y. Chen, C.-Z. Lo, C. A. Hsiung, hp-dpi: Helicobacter pylori database of protein interactomesembracing experimental and inferred interactions, Bioinformatics 21 (7) (2005) 1288-1290. 
[49] G. Butland, J. M. Peregrín-Alvarez, J. Li, W. Yang, X. Yang, V. Canadien, A. Starostine, D. Richards, B. Beattie, N. Krogan, et al., Interaction network containing conserved and essential protein complexes in escherichia coli, Nature 433 (7025) (2005) 531-537.

[50] P. Noirot, M.-F. Noirot-Gros, Protein interaction networks in bacteria, Current opinion in microbiology 7 (5) (2004) 505-512.

[51] R. Milo, S. Itzkovitz, N. Kashtan, R. Levitt, S. Shen-Orr, I. Ayzenshtat, M. Sheffer, U. Alon, Superfamilies of evolved and designed networks, Science 303 (5663) (2004) $1538-1542$.

[52] G. F. Davis, M. Yoo, W. E. Baker, The small world of the american corporate elite, 1982-2001, Strategic organization 1 (3) (2003) 301-326.

[53] D. MacRae, Direct factor analysis of sociometric data, Sociometry 23 (4) (1960) 360-371.

[54] Data from nih grants da12831 and hd41877 (2001).

[55] W. W. Zachary, An information flow model for conflict and fission in small groups, Journal of anthropological research 33 (4) (1977) 452-473.

[56] L. D. Zeleny, Adaptation of research findings in social leadership to college classroom procedures, Sociometry 13 (4) (1950) 314-328.

[57] D. Krackhardt, The ties that torture: Simmelian tie analysis in organizations, Research in the Sociology of Organizations 16 (1) (1999) 183-210.

[58] W. De Nooy, A. Mrvar, V. Batagelj, Exploratory social network analysis with Pajek, Cambridge University Press, 2018.

[59] J. H. Michael, J. G. Massey, Modeling the communication network in a sawmill, Forest Products Journal 47 (9) (1997) 25.

[60] C. R. Myers, Software systems as complex networks: Structure, function, and evolvability of software collaboration graphs, Physical Review E 68 (4) (2003) 046116. 
[61] D. J. Watts, S. H. Strogatz, Collective dynamics of small-worldnetworks, nature 393 (6684) (1998) 440.

[62] D. Plavšić, S. Nikolić, N. Trinajstić, Z. Mihalić, On the harary index for the characterization of chemical graphs, Journal of Mathematical Chemistry 12 (1) (1993) 235-250.

[63] V. Latora, M. Marchiori, Efficient behavior of small-world networks, Physical review letters 87 (19) (2001) 198701.

[64] E. Estrada, The communicability distance in graphs, Linear Algebra and its Applications 436 (11) (2012) 4317-4328.

[65] E. Estrada, Complex networks in the euclidean space of communicability distances, Physical Review E 85 (6) (2012) 066122.

[66] D. J. Klein, M. Randić, Resistance distance, Journal of mathematical chemistry 12 (1) (1993) 81-95.

[67] E. Estrada, N. Hatano, Communicability angle and the spatial efficiency of networks, SIAM Review 58 (4) (2016) 692-715.

[68] R. Boese, H.-C. Weiss, D. Bläser, The melting point alternation in the short-chain nalkanes: single-crystal x-ray analyses of propane at $30 \mathrm{k}$ and of n-butane to n-nonane at 90 k, Angewandte Chemie International Edition 38 (7) (1999) 988-992.

[69] L. C. Freeman, Centrality in social networks conceptual clarification, Social networks 1 (3) (1978) 215-239.

[70] L. C. Freeman, A set of measures of centrality based on betweenness, Sociometry (1977) $35-41$.

[71] P. Bonacich, Power and centrality: A family of measures, American journal of sociology 92 (5) (1987) 1170-1182.

[72] E. Estrada, J. A. Rodriguez-Velazquez, Subgraph centrality in complex networks, Physical Review E 71 (5) (2005) 056103. 